\title{
Optimal resource allocation in a representative investor economy is
}

\author{
Orlando Gomes * \\ Lisbon Accounting and Business School (ISCAL/IPL), Business Research Unit, (UNIDE/ISCTE-IUL), Portugal
}

\section{A R T I C L E I N F O}

Article history:

Accepted 16 June 2015

Available online 2 July 2015

\section{Keywords:}

Representative investor

Agency problem

Optimal control

Saddle-path stability

Neoclassical growth model

AK endogenous growth model

Sentiment shocks

\begin{abstract}
A B S T R A C T
The Ramsey-Cass-Koopmans neoclassical growth setup and the AK endogenous growth framework are two prototypes of a class of growth models where, by assumption, investment resources are always perfectly allocated to production. As a corollary, these models offer a partial view of the growth process circumscribed to a limit case, namely the most favorable case in which all possible frictions on the allocation of investment resources are absent. This paper adds to the conventional growth setup an optimal mechanism of assignment of investment resources that contemplates the possibility of inefficient allocation. In the assumed economy there is a single representative investor and a large number of firms that compete for the available resources. The new setup highlights how agency costs may deviate the economy from the benchmark growth outcome, potentially generating less desirable long-run results. The appeal of the proposed framework resides also on the fact that new determinants of growth emerge and take a leading role, namely the investor's sentiment and the quality of the firms' investment proposals.
\end{abstract}

(c) 2015 Elsevier B.V. All rights reserved.

\section{Introduction}

Benchmark growth models offer a partial view on the process of wealth creation. In such models, no friction on the allocation of investment is present and, therefore, the economy tends to an upper limit outcome of full efficiency. This paper devises and explores the dynamics of a stylized optimal control problem concerning the intertemporal allocation of investment across firms in a market economy which, once merged with neoclassical and endogenous growth setups, allows to gain a wider perspective on the growth process as approached by standard theory in this field of knowledge.

The introduction of a mechanism inducing inefficiency on investment allocation is relevant from an economic growth point of view because it allows to depart from the implicit setting of full employment of resources. Departures from potential output are now contemplated, and they will be determined by a multiplicity of factors, that might even include animal spirits under the form of the investor's sentiment. As one will realize, the new structure of analysis is much more amenable to the introduction of a discussion on the compatibility between business cycles and growth than the conventional growth models.

The proposed setup involves a single representative investor who decides, based on criteria established at the initial date, how to assign,

\footnotetext{
it I acknowledge the insightful and helpful comments of an anonymous referee and of the journal's editor. The usual disclaimer applies. I also thank the financial support of the Business Research Unit of the Lisbon University Institute (Grant code: PEst-OE/EGE/ UI0315/2011).

* Lisbon Accounting and Business School (ISCAL/IPL), Av. Miguel Bombarda 20, 1069035 Lisbon, Portugal.

E-mail address: omgomes@iscal.ipl.pt.
}

over time, a given amount of financial resources. Firms, that exist in the economy in a potentially large number, will compete for those resources through the presentation to the investor of projects that detail the activities they will engage on.

In the described setting, the investment allocation problem acquires the nature of an agency problem, where the representative investor plays the role of principal and the firms are the agents. Because the investor does not know with anticipation how the assigned budget will be employed by each firm, the criteria used to allocate it is the signaling effort of each firm; firms communicate their financial needs to the investor through the presentation of projects that require resources to be prepared.

The allocation of funds involves a matching function between the quality of the mentioned project proposals and the funds that remain available to be attributed. This matching is such that both inputs are essential to obtain a positive outcome: at each specific time period, no financial transfer will occur if the firm under consideration does not present any investment project proposal; likewise, if no funds remain available, no matter the quality of the proposed projects, the investment transfer will not take place. Furthermore, in the model's setting, it is considered that at each period the investor claims back from every firm a constant percentage of the previously allocated funds, e.g., under the form of contracted capital returns. This forces the firms to systematically present new projects since, otherwise, they will progressively lose the financial resources obtained in the past, and their funding will end up by falling to zero.

The investment allocation problem will be analyzed from the point of view of the firm. The firm will want to maximize, on an intertemporal perspective, the difference between the financial resources it is capable 
of obtaining and the costs it incurs to convince the investor about the merits of its investment plan. This optimization problem will be subject to a budget flow constraint, where the firm's assigned budget increases with the already mentioned matching process and diminishes with the automatic withdrawal of funds at a constant rate.

Although simple in its structure, this is an analytically tractable model that delivers relevant insights on the process of investment allocation. Given the signaling costs, this model conducts to a result of inefficiency, in the sense that, in a long-term perspective, the aggregate allocated investment will remain below $100 \%$. A unique steady-state point is determined and its position depends decisively on two entities: the quality of the projects presented by the firms and the overall sentiment of the investor. As these forces shape the amount of aggregate investment, they will also be main determinants of economic growth.

Project quality and investor sentiment are, according to the available empirical evidence, two important drivers of the overall aggregate investment level and of the ability of the economies to generate additional wealth. Section 2.2 briefly reviews some of the empirical literature that highlights the key role that each of the two mentioned features has in what concerns observed growth patterns. This evidence is the key motivation for setting up the growth model in the sections that follow. By placing investment in the forefront of the growth analysis one is able to realistically approach long-run wealth accumulation, without neglecting short-term fluctuations due to changes in the determinants of investment levels.

The remainder of the paper is organized as follows. In Section 2, a review of the relevant related literature is undertaken; this review covers both theoretical contributions and empirical studies that highlight project quality and investor's sentiment as main drivers of the growth process. Section 3 makes the analytical presentation of the problem of the firm. Section 4 derives optimality conditions and discusses the existence of the steady-state. In Section 5, the stability of the longterm equilibrium is investigated. Section 6 studies how eventual changes on the values of exogenous variables, including the investor's sentiment, might disturb transitional dynamics and the steady-state. In Section 7, the analytical framework of this paper is merged with the Ramsey-Cass-Koopmans model in order to address the respective implications for growth, in a neoclassical perspective. Section 8 adapts the dynamic resource allocation problem to a setting of endogenous growth. Finally, Section 9 concludes.

\section{Related literature}

\subsection{Theoretical foundations}

The analysis in the following sections is inspired by a confluence of different strands of literature in economics. First, the financial literature that highlights agency relations and other sources of conflict between investors and investment recipients, which typically lead to investment distortions. Although the studies in turn of this subject tend to be circumscribed to a unique firm or group of integrated firms that undertake diversified activities, with the managers within each department or division negotiating with the shareholders the corresponding budget allocations, such discussion is straightforward to extrapolate to our environment of a unique representative investor in the economy. About agency and power relations within the firm, with consequences on the allocation of scarce resources see, e.g., Shleifer and Vishny (1989), La Porta et al. (2000), Rajan et al. (2000), Burkart and Panunzi (2006), Agliardi and Agliardi (2008), Albuquerque and Wang (2008) and Chetty and Saez (2010).

The second relevant line of inquiry relates to the extensive literature that searches for an integrated analysis of growth and business cycles. A significant part of this literature takes conventional growth models and explores the mechanisms that may induce persistent deviations of varying amplitude relatively to the full rational/full efficient benchmark outcome. One notorious contribution in this field is the work by Evans et al. (1998), who identify the possibility of formation of two different balanced growth paths, one respecting to a state of low growth and the other to a state of high growth. The economy may converge to one or the other steady-state, depending on the agents' expectations.

Other work on growth cycles puts R\&D investment at the center of the discussion. It is the case of Matsuyama (1999, 2001), Eriksson and Lindh (2000), Walde (2002, 2005), Francois and Lloyd-Ellis (2003) and Shinagawa (2013). These studies emphasize the role of the cyclical behavior of the investment in innovation, in some cases triggered by stochastic shocks and in other cases by endogenous processes, in shaping long-term cyclical growth.

The search for endemic causes of deterministic growth cycles has a long tradition in economics, starting in the 1980s with the work of Benhabib and Day (1981), Day (1982), Grandmont (1985) and Deneckere and Pelikan (1986), among others. Meaningful contributions on the field of endogenous growth cycles include Christiano and Harrison (1999), who proved the existence of chaos in a standard deterministic version of the Real Business Cycles (RBC) model with production externalities, Brock and Hommes (1997, 1998), who encountered rational routes to randomness in a setting of heterogeneous expectations and discrete choice across distinct deliberation rules, Boldrin et al. (2001), who address the conditions under which endogenous growth models might generate endogenous cyclical motion, Gomes (2008), who considers that the non-rival nature of technology conflicts with congestion externalities triggering in this way endogenous fluctuations, and Drugeon (2013), who highlights how a standard growth model with heterogeneous capital goods may conduct to a result that departs from stability and give rise to competitive equilibrium growth cycles that are optimally generated.

In this article, growth cycles are not explicitly modeled, but the offered framework creates the setting that allows to analyze their presence. By conceiving an environment where optimal decisions by rational agents originate a less than efficient equilibrium result, in the sense that part of the investment resources will remain unemployed, one will be able to approach the conditions that lead to low or high growth (i.e., growth results that depart more or less from the full efficiency benchmark outcome). If one allows for multiple investor's sentiment states or systematic changes on the quality level of the projects presented by firms to the investor, then one will be able to approach cyclical motion in the presented growth framework.

A third strand of thought with relevance to the study in this paper concerns the resurgence of animal spirits as a main determinant of the explanation of short-run aggregate fluctuations. In a typical growth model, the entities capable of disturbing the generated balanced growth path are technology, the preferences of consumers and other fundamentals. RBC theory is built precisely on this assumption: systematic changes on the technical conditions of production and other supply side effects are, under this interpretation, the determinants of observed fluctuations (see King and Rebelo, 1999; Rebelo, 2005).

Alternative explanations, namely those that place the emphasis on agents' sentiments or animal spirits, have occasionally appeared in the literature. The most popular of these approaches is the one relating multiple equilibria and indeterminacy in optimal growth models. The indeterminacy or sunspots literature, developed by Azariadis (1981), Cass and Shell (1983), Benhabib and Farmer (1994), Weder (2004) and Guerrazzi (2012), among others, supports that self-fulfilling prophecies leading to fluctuations might emerge in frictionless economies when the neoclassical model is modified in order to display multiple equilibria. Under multiple equilibria, the beliefs of agents determine the trajectory to be followed from a series of optimal trajectories generated by economic fundamentals. Indeterminacy implies that there is not a unique optimal path to follow and, in this case, the trajectory that is effectively followed depends on the self-fulfilling beliefs of the agents.

A revival of animal spirits as a centerpiece of the macroeconomic thought occurred with the publication of the book by Akerlof and Shiller (2009), which coincided with the great recession of the end of 
the first decade of the twenty-first century. In the book, the view that individual sentiments matter for economic outcomes was emphasized, leading to a new wave of interest on the impact of agents' sentiments over economic results.

In the work that followed, two studies deserve to be highlighted. First, De Grauwe (2011), who claims that rational expectations and animal spirits do not mix well and, thus, considers the presence of cognitive deficiencies in the way individuals process information. This does not mean that agents are irrational; instead, they are rational in understanding that they have a limited capacity to interpret reality and, therefore, have to use simple heuristics to take decisions. De Grauwe's model generates endogenous waves of optimism and pessimism that might be associated to the notion of animal spirits.

On a different plan, Angeletos and La'O (2013) propose a framework of analysis that combines market sentiments or animal spirits with the paradigm of rational expectations, as in the sunspots analysis. Differently from the sunspots setup, though, in the mentioned article multiple equilibria are absent. The sentiments of agents emerge as the outcome of communication constraints. The authors prove that if the communication is random and decentralized, business cycles might arise in the context of a typical neoclassical model, independently of shocks to fundamentals. It is the propagation of information that generates, in this case, endogenous fluctuations based on waves of optimism and pessimism.

In our article, there are evident departures relatively to the previous contributions, but a relevant role is, in the same way, assigned to sentiments, namely investor sentiments. Shocks on the confidence level of the representative investor will imply different rates of utilization of the resources available to generate value, implying potential fluctuations on the level of aggregate output.

In what concerns the particular modeling apparatus used in this paper, it has antecedents on Gomes $(2014,2015)$, where inefficiency due to agency relations is applied, respectively, to resource allocation in the brain and consequent cognitive decisions of the individual agent, and to a problem of budget setting within the divisions of a given firm.

\subsection{A remark on the empirics of project quality and investor's sentiment}

In the introduction, it was highlighted that the quality of investment projects and the investor's sentiment will be the main drivers of growth in the analysis to pursue in the sections that follow. Besides this being an intuitive fact, there is also plenty evidence in the economics literature pointing to the relevance of these elements as forces that decisively shape long-term growth trajectories.

Concerning project quality, one should start by noting that this is not a concept completely void of ambiguity. From a strictly financial point of view, the feasibility of a project is measured by its return in terms of expected cash-flows, but many other dimensions are relevant to assess investment quality from an economic growth point of view; the environmental impact or the expected society-wide externalities, positive and negative, for instance, are two features that must be taken into account as well.

Independently of the considered definition, the idea that the quality of investment projects is a key determinant of growth finds in the empirical literature strong substantiation. This is, for instance, the result pointed out by Sala-i-Martin and Artadi (2002), who have assessed the growth performance of Middle-East countries. The authors claim that the persistent low growth in this region of the globe is the direct consequence of wrong or inefficient investment choices, which have their profound roots on several infra-structural deficiencies, namely the poor quality of institutions (financial, political, educational and others).

The discussion on the quality of investment projects is most of the times associated with, on one hand, public investment and, on the other hand, foreign direct investment. Gupta et al. (2014) and O'Toole and Tarp (2014) address the issue of public investment efficiency, mainly in the perspective of the development of low-income economies. The mentioned studies stress that measures of the adequacy of public investment projects are a statistically significant variable when explaining growth in developing countries. Furthermore, public authorities should be concerned with suppressing rent-seeking behavior, bureaucracy and corruption, since these are sources of inefficiency in the allocation of capital, leading to poor quality projects, and thus impair growth.

The notion of investment quality is also present on how the impact of capital flows from abroad is assessed. Alfaro and Charlton (2007) highlight the idea that some types of foreign direct investment are preferable than others; these authors associate the notion of foreign investment quality to the effect that such investment has on growth in the host country. In the same vein, Beckaert et al. (2011) demonstrate that the openness to capital flows from abroad typically enhances investment efficiency and increases the quality of the investment projects in the economy; and this is positively and strongly correlated with aggregate output growth.

A second strong claim in our analysis is that the investor's sentiment is a main driver of the aggregate investment flows that determine the pace of growth. This is documented, for instance, in the study by Arif and Lee (2014), who evaluate the relation between the sentiment of investors, the expected equity returns and the level of aggregate investment; not surprisingly, they conclude that periods when sentiments of optimism dominate are also periods in which the investment of firms reaches the highest observable levels. Montone and Zwinkels (2015) also arrive to the conclusion that investor sentiments have real effects; specifically, these authors discuss the correlation between the sentiment of capital owners and the growth of employment and output.

Another piece of evidence on the relation between investor confidence and growth is provided by Milani (2014), who estimates a macroeconomic general equilibrium model and finds evidence that psychological factors, namely those related to investment decisions, are a main determinant of the observable aggregate business cycles (they are responsible, according to this study, by more than $40 \%$ of the observable business fluctuations, in the United States).

\section{The dynamic resource allocation problem}

Consider an economy populated by a single representative investor and a large number of firms, each one dedicated to the development of a specific productive task $i \in N .{ }^{1}$ Firms compete for the available investment resources, which the representative investor allocates, at each date $t \geq 0$, after evaluating two criteria: (i) the signaling effort of each firm and (ii) the investment resources that remain available to assign.

Two arrays of endogenous variables play a role in the model: the investment shares allocated to the activities of each firm, $\omega_{i}(t)$, and the costs firms incur when presenting their projects to the capital holder, $\phi_{i}(t)$, which are also measured as percentages of the available investor's budget. Variables $\omega_{i}(t)$ and $\phi_{i}(t)$ correspond to non-negative values $\forall t \geq 0$, and the respective aggregate levels cannot assume values larger than the unity, i.e., $\Omega(t) \equiv \int_{i \in N} \omega_{i}(t) d i \leq 1$ and $\Phi(t) \equiv \int_{i \in N} \phi_{i}(t) d i \leq 1$.

The objective of each firm is to maximize, in an intertemporal perspective, the difference between the collected amount of investment and the signaling costs associated with their acquisition. In this setting, variable $\phi_{i}(t)$ acquires the role of control variable, i.e., the firm is free to choose how much it commits to acquire the funds that will serve the development of productive activities, in order to fulfill the referred

\footnotetext{
${ }^{1}$ Although firms develop different tasks, we consider these to be perfect substitutes and, thus, a perfectly competitive market environment is considered. This assumption is useful later on the paper, when addressing the impact of inefficient investment allocation over growth. Alternatively, a monopolistic competition setup could be taken, if the productive tasks developed by firms presented some degree of substitutability. This would imply considering a Dixit-Stiglitz production function when presenting the growth framework, instead of the Cobb-Douglas function that will be assumed. There are no meaningful differences, in terms of the model's implications, of assuming one or the other specification.
} 
objective. Under an infinite horizon and taking a constant in time discount rate, $\rho>0$, the objective function of firm $i$ is, ${ }^{2}$

$U_{i}(0)=\operatorname{Max}_{\phi_{i}(t)} \int_{0}^{+\infty}\left[\omega_{i}(t)-\phi_{i}(t)\right] \exp (-\rho t) d t, \quad i \in N$.

A law of motion that is beyond the control of each firm will determine the evolution over time of the assigned investment share, $\omega_{i}(t)$; this is the state variable of the problem. Two processes work in opposite directions. The first process, through which the allocated investment share potentially increases, is generated by a matching function that puts into confrontation the firm's signaling effort and the resources the investor has left to be allocated at date $t$.

\section{Definition 1. Matching function}

Function $f_{i}\left[\phi_{i}(t), 1-\Omega(t)\right]: \mathbb{R}_{+}^{2} \rightarrow \mathbb{R}_{+}$is a matching function of the firm's dynamic investment allocation problem if it obeys the following properties:

i) $f_{i}$ is a continuous and differentiable function;

ii) $f_{i}$ is increasing in both arguments: $\partial f_{i} / \partial \phi_{i}>0, \partial f_{i} / \partial(1-\Omega)>0$;

iii) $f_{i}$ is subject to decreasing marginal returns: $\partial^{2} f_{i} / \partial \phi_{i}^{2}<0$, $\partial^{2} f_{i} / \partial(1-\Omega)^{2}<0$

iv) $f_{i}$ is homogeneous of degree $1: f_{i}\left[\epsilon \phi_{i}(t), \epsilon(1-\Omega(t))\right]=$ $\epsilon f_{i}\left[\phi_{i}(t), 1-\Omega(t)\right], \forall \epsilon>0$;

$v)$ Both inputs are essential for the matching process, $f_{i}[0,1-\Omega(t)]=f_{i}\left[\phi_{i}(t), 0\right]=0$.

A possible explicit functional form for the matching function, that obeys the above properties, is a Cobb-Douglas function of the type,

$f_{i}\left[\phi_{i}(t), 1-\Omega(t)\right]=B\left[q_{i} \phi_{i}(t)\right]^{\beta}[1-\Omega(t)]^{1-\beta}, \quad i \in N, B>0, q_{i}>0, \beta \in(0,1)$.

Three parameters play a role in function (2); $\beta$ is an elasticity parameter, $B$ is a measure of the investor's sentiment (a stronger positive sentiment implies a more efficient matching), and $q_{i}$ represents the quality of the projects firm $i$ proposes to the investor. The quality parameter is not necessarily of a same value across firms, meaning that they are heterogeneous regarding the ability to convince the investor in financing the respective activities. Note that parameter $q_{i}$ emerges in the matching function as a weight over the signaling costs; this signifies that the input of the matching function is not just the cost incurred by the firm to communicate investment plans; the effectiveness of the communication is relevant as well.

The second force shaping the pace of the investment share respects to an automatic withdrawal of resources by the investor, who claims back, at each time period, a constant percentage of the attributed funds. This allows the investor to continuously hold financial resources in search for the best investment opportunities, and compels firms, in order to maintain or increase their budgets, to present, systematically over time, new projects to the investor. If no effort is made by the firm to acquire resources, i.e., if $\phi_{i}(t)=0$, then the firm will lose allocated resources at a rate $\zeta \in(0,1)$. form $^{3}$

Under the specified conditions, the resource constraint takes the $\dot{\omega}_{i}(t)=f_{i}\left[\phi_{i}(t), 1-\Omega(t)\right]-\zeta \omega_{i}(t), \quad i \in N, \omega_{i}(0)=\omega_{i 0} \in(0,1)$ given.

\footnotetext{
2 The discount rate indicates the degree of impatience of firm $i$ concerning future net budget allocation. A value of $\rho$ close to zero implies a low level of impatience; if $\rho$ is high, the future is heavily discounted and the agent is highly impatient. In order to simplify the analysis, one considers that the discount rate is identical across firms.

${ }^{3}$ Note that the model's formulation is purely deterministic and that perfect foresight holds for every assumed dynamic relation.
}

\section{Definition 2. DRAP}

The dynamic resource allocation problem (DRAP) faced by firm $i$ consists in solving Eq. (1) subject to Eq. (3) and to the constraints on variables $\omega_{i}(t) \geq 0, \phi_{i}(t) \geq 0$, and $\Omega(t) \leq 1, \Phi(t) \leq 1$.

\section{Optimal solution and the steady-state}

The solution of the DRAP will be a system of dynamic equations reflecting the motion of the investment shares and of the respective co-state variable, $p_{i}(t) \geq 0$.

Proposition 1. The optimal solution of the DRAP, for the Cobb-Douglas matching function, corresponds to the system of differential equations

$$
\begin{aligned}
\dot{\omega}_{i}(t)= & B^{1 /(1-\beta)}\left[\beta q_{i} p_{i}(t)\right]^{\beta /(1-\beta)}[1-\Omega(t)]-\zeta \omega_{i}(t), \quad i \in N \\
\dot{p}_{i}(t)= & (\rho+\zeta) p_{i}(t)-1 \\
& +(1-\beta) \beta^{\beta /(1-\beta)} B^{1 /(1-\beta)} \int_{j \in N}\left[q_{j}^{\beta /(1-\beta)} p_{j}(t)^{1 /(1-\beta)}\right] d j, \quad i \in N .
\end{aligned}
$$

Proof. The current-value Hamiltonian function of the optimal control DRAP is, for a firm $i$,

$$
\begin{aligned}
H\left[\phi_{i}(t), \omega_{i}(t), p_{i}(t)\right]= & \omega_{i}(t)-\phi_{i}(t) \\
& +\int_{j \in N} p_{j}(t)\left\{B\left[q_{j} \phi_{j}(t)\right]^{\beta}[1-\Omega(t)]^{1-\beta}-\zeta \omega_{j}(t)\right\} d j, \quad i \in N .
\end{aligned}
$$

Applying Pontryagin's principle, the computation of first-order optimality conditions yields, ${ }^{4}$

$$
\begin{aligned}
& \frac{\partial H}{\partial \phi_{i}(t)}=0 \Rightarrow \phi_{i}(t)=q_{i}^{\beta /(1-\beta)}\left[\beta B p_{i}(t)\right]^{1 /(1-\beta)}[1-\Omega(t)], \quad i \in N \\
& \dot{p}_{i}(t)=\rho p_{i}(t)-\frac{\partial H}{\partial \omega_{i}(t)} \Rightarrow \\
& \dot{p}_{i}(t)=(\rho+\zeta) p_{i}(t)-1+(1-\beta) B \int_{j \in N}\left\{p_{j}(t)\left[\frac{q_{j} \phi_{j}(t)}{1-\Omega(t)}\right]^{\beta}\right\} d j, i \in N
\end{aligned}
$$

and the transversality condition for infinite horizon problems, which is, in this case,

$\lim _{t \rightarrow+\infty} p_{i}(t) \exp (-\rho t) \omega_{i}(t)=0, \quad i \in N$.

To determine the rule of motion of the co-state variable, Eq. (5), just replace $\phi_{i}(t)$, as defined by Eq. (7), into the first-order condition Eq. (8):

$$
\begin{aligned}
\dot{p}_{i}(t)= & (\rho+\zeta) p_{i}(t)-1 \\
& +(1-\beta) B \int_{j \in N}\left\{p_{j}(t)\left[\frac{q_{j}\left\{q_{j}^{\beta /(1-\beta)}\left[\beta B p_{j}(t)\right]^{1 /(1-\beta)}[1-\Omega(t)]\right\}}{1-\Omega(t)}\right]\right\} d j, i \in N \Leftrightarrow \\
\dot{p}_{i}(t)= & (\rho+\zeta) p_{i}(t)-1+(1-\beta) B \int_{j \in N}\left\{p_{j}(t)\left[\beta B q_{j} p_{j}(t)\right]^{\beta /(1-\beta)}\right\} d j, i \in N \Leftrightarrow \\
\dot{p}_{i}(t)= & (\rho+\zeta) p_{i}(t)-1+(1-\beta) \beta^{\beta /(1-\beta)} B^{1 /(1-\beta)} \int_{j \in N}\left[q_{j}^{\beta /(1-\beta)} p_{j}(t)^{1 /(1-\beta)}\right] d j, i \in N .
\end{aligned}
$$

\footnotetext{
4 The optimality conditions hold under the presumption that the constraints over variables $\omega_{i}(t) \geq 0, \phi_{i}(t) \geq 0$, and $\Omega(t) \leq 1, \Phi(t) \leq 1$ are not violated. As the analytical study unfolds, one will confirm that the constraints are satisfied for any $t \geq 0$, whenever the following set of conditions apply:

(i) The initial state of the system locates in the vicinity of the system's steady-state;

(ii) $\Omega(0)<\Omega^{*}$, with $\Omega^{*}$ the steady-state value of $\Omega(t)$;

(iii) The steady-state value of the co-state variable, $p^{*}$, obeys constraint $\frac{2 \beta-1}{\beta} \frac{1}{\rho+\zeta} \leq p^{*}<$ $\frac{1}{\rho+\zeta}$ if $\beta>\frac{1}{2}$ or $0<p^{*}<\frac{1}{\rho+\zeta}$ if $\beta \leq \frac{1}{2}$.
} 
The other differential equation of the system, Eq. (4), is obtained by substituting $\phi_{i}(t)$, as in Eq. (7), into the constraint Eq. (3):

$$
\begin{aligned}
& \dot{\omega}_{i}(t)=B\left[q_{i}\left\{q_{i}^{\beta /(1-\beta)}\left[\beta B p_{i}(t)\right]^{1 /(1-\beta)}[1-\Omega(t)]\right\}\right]^{\beta}[1-\Omega(t)]^{1-\beta}-\zeta \omega_{i}(t) \Leftrightarrow \\
& \dot{\omega}_{i}(t)=B\left[\beta B q_{i} p_{i}(t)\right]^{\beta /(1-\beta)}[1-\Omega(t)]-\zeta \omega_{i}(t) \Leftrightarrow \\
& \dot{\omega}_{i}(t)=B^{1 /(1-\beta)}\left[\beta q_{i} p_{i}(t)\right]^{\beta /(1-\beta)}[1-\Omega(t)]-\zeta \omega_{i}(t) .
\end{aligned}
$$

The pair of differential Eqs. (4)-(5) is a deterministic twodimensional dynamic system with two endogenous variables. A first step in the process of evaluating this system's dynamics consists in assessing the properties of the steady-state.

Definition 3. Steady-state

The steady-state of the DRAP is the point $\left(\omega_{i}^{*}, p_{i}^{*}\right)=\left\{\left(\omega_{i}^{*}, p_{i}^{*}\right): \dot{\omega}_{i}(t)=\right.$ $\left.0, \dot{p}_{i}(t)=0, i \in N\right\}$.

Given the definition of the steady-state for the problem under consideration, the following result is accomplished,

Proposition 2. The steady-state point of the DRAP, $\left(\omega_{i}^{*}, p_{i}^{*}\right), i \in N$, exists and it is unique.

Proof. Applying condition $\dot{p}_{i}(t)=0$ to equation of motion Eq. (5),

$$
(1-\beta) \beta^{\beta /(1-\beta)} B^{1 /(1-\beta)} \int_{j \in N}\left[q_{j}^{\beta /(1-\beta)}\left(p_{j}^{*}\right)^{1 /(1-\beta)}\right] d j=1-(\rho+\zeta) p_{i}^{*} .
$$

Observe that the lhs of expression Eq. (10) is the same independently of the firm for which the optimality problem is approached. An immediate corollary of this observation is that $p_{i}^{*}=p_{j}^{*}, \forall i, j \in N$. In the light of this result, define $p^{*} \equiv p_{i}^{*}, \forall i \in N$, and rewrite Eq. (10) as

$$
(1-\beta) \beta^{\beta /(1-\beta)}\left(B p^{*}\right)^{1 /(1-\beta)} \int_{j \in N} q_{j}^{\beta /(1-\beta)} d j=1-(\rho+\zeta) p^{*}
$$

Expression Eq. (11) does not allow for the determination of an explicit solution for the steady-state level of the co-state variable. However, it is straightforward to verify that this steady-state value exists and is unique. Begin by observing that the rhs of Eq. (11) is a straight line starting at 1 (for $p^{*}=0$ ) and possessing a negative slope; while the lhs is an increasing convex function of $p^{*}$ starting at 0 (for $p^{*}=0$ ). Given the shape of the two mentioned functions, they will intersect in a single point for $p \in\left(0, \frac{1}{\rho+\zeta}\right)$.

To complete the proof, one needs to demonstrate that $\omega_{i}^{*}$ exists and is unique as well. Solving $\dot{\omega}_{i}(t)=0$, given Eq. (4), the following relation emerges,

$$
1-\Omega^{*}=\frac{\zeta \omega_{i}^{*}}{B^{1 /(1-\beta)}\left(\beta q_{i} p_{i}^{*}\right)^{\beta /(1-\beta)}}
$$

with $\Omega^{*} \equiv \int_{j \in N} \omega_{j}^{*} d j$. Because the steady-state value of the co-state variable is identical across firms, Eq. (12) implies that, for any $i, j \in N$,

$\frac{\omega_{i}^{*}}{\omega_{j}^{*}}=\left(\frac{q_{i}}{q_{j}}\right)^{\beta /(1-\beta)}$.

Relation Eq. (13) allows for rewriting $\Omega^{*}$, given the respective definition,

$\Omega^{*}=\frac{1}{q_{i}^{\beta /(1-\beta)}} \int_{j \in N} q_{j}^{\beta /(1-\beta)} d j \omega_{i}^{*}$.
Replacing $\Omega^{*}$, as displayed in Eq. (14), into Eq. (12), the steady-state of variable $\omega_{i}(t)$ is given as

$\omega_{i}^{*}=\frac{B^{1 /(1-\beta)}\left(\beta q_{i} p^{*}\right)^{\beta /(1-\beta)}}{B^{1 /(1-\beta)}\left(\beta p^{*}\right)^{\beta /(1-\beta)} \int_{j \in N} q_{j}^{\beta /(1-\beta)} d j+\zeta}, i \in N$.

Since there is a unique $p^{*}$, expression Eq. (15) guarantees that a unique $\omega_{i}^{*}$ exists as well. Nevertheless, note that $\omega_{i}^{*}$ is different across firms, given the different quality of the projects presented to the investor.

Several elements in the proof of Proposition 2 deserve a comment. First, one can visualize the steady-state outcome for the co-state variable through the graphical representation of the lhs and of the rhs of Eq. (10). The intersection point delivers $p^{*}$ as depicted in Fig. 1.

Second, note that the mechanics of the model impose an indisputable upper bound on the value of $p^{*}$; one has stated, in Footnote 4, that this upper bound is required to guarantee that boundaries on the values of variables are respected. In fact, such upper bound does not need to be forced into the model, since it arises spontaneously. One will get back to this issue later when directly exploring the feasibility conditions of the model. A third point relates to Eq. (13). One should highlight that it delivers a relevant result: it states that in the long term steady-state the single determinant of the difference between investment shares allocated across firms is the quality of project signaling.

A last remark is associated with steady-state result (Eq. (15)). One can use this to obtain the expression of the aggregate investment share, also evaluated in the steady-state. Summing across all $\omega_{i}^{*}$,

$\Omega^{*}=\frac{B^{1 /(1-\beta)}\left(\beta p^{*}\right)^{\beta /(1-\beta)} \int_{i \in N} q_{i}^{\beta /(1-\beta)} d i}{B^{1 /(1-\beta)}\left(\beta p^{*}\right)^{\beta /(1-\beta)} \int_{i \in N} q_{i}^{\beta /(1-\beta)} d i+\zeta}$.

Share $\Omega^{*}$ can be represented, in an alternative form, once one combines Eqs. (16) and (11),

$\Omega^{*}=\frac{1-(\rho+\zeta) p^{*}}{1-(\rho+\beta \zeta) p^{*}}$

It is evident, both from Eqs. (16) and (17), that $\Omega^{*}<1$. This result is important because it indicates that no matter the efficiency of the investment allocation process, the agency relation that is established between the investor and the firms will prevent investment resources to be fully allocated. Naturally, allocation results improve with a better quality of the investment project proposals or with a more optimistic investor sentiment, as one will confirm with the formal analysis in Section 6; however, independently of the values of $B$ and $q_{i}$, there will

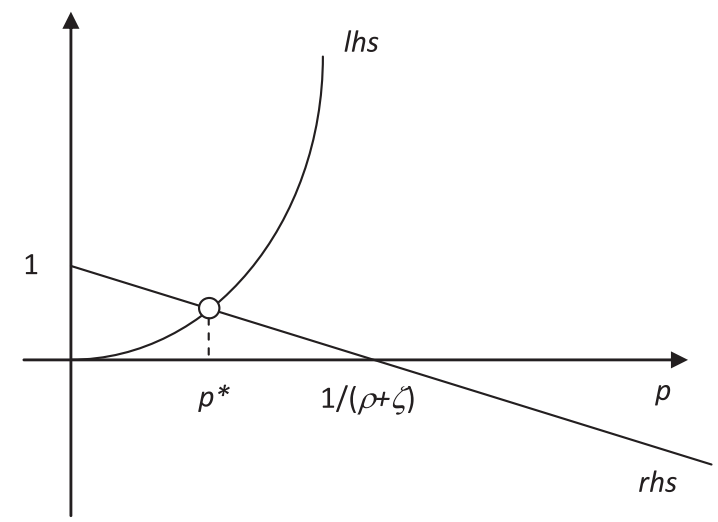

Fig. 1. Steady-state uniqueness for the co-state variable. 
always be some waste of investment resources if they are allocated under the mechanics of the proposed dynamic model.

Another steady-state result is the one respecting the control variable. The evaluation of Eq. (7) in the steady-state, given the relation between $p^{*}$ and $\Omega^{*}$ in expression Eq. (16), yields

$\phi_{i}^{*}=\frac{(1-\beta) \zeta(\beta B)^{1 /(1-\beta)}\left(p^{*}\right)^{(2-\beta) /(1-\beta)} q_{i}^{\beta /(1-\beta)}}{1-(\rho+\beta \zeta) p^{*}}$.

Note that, according to Eq. (18), the steady-state of the signaling cost variable differs across firms, given the difference in the quality of the proposed investment projects. For any $i, j \in N$,

$\frac{\phi_{i}^{*}}{\phi_{j}^{*}}=\left(\frac{q_{i}}{q_{j}}\right)^{\beta /(1-\beta)}$.

As for the budget share variable, the steady-state signaling cost incurred by firm $i$ relatively to the signaling cost incurred by firm $j$ is as much larger as the wider is the difference in terms of the quality of the investment projects.

The steady-state value of the aggregate level of the signaling costs across firms is obtainable directly from Eq. (18),

$\Phi^{*}=\int_{i \in N} \phi_{i}^{*} d i=\beta \zeta p^{*} \Omega^{*}$

\section{Local stability}

In this section, transitional dynamics of the DRAP are evaluated. A point $\left(\omega_{i 0}, p_{i 0}\right)$ in the vicinity of $\left(\omega_{i}^{*}, p_{i}^{*}\right)$ is considered and local stability is approached through the linearization of the system of Eqs. (4)-(5). The linearization process requires the computation of the corresponding Jacobian matrix, which can be partitioned in four distinct blocks,

$J=\left[\begin{array}{ll}J_{11} & J_{12} \\ J_{21} & J_{22}\end{array}\right]$

Matrix $J_{m n}, m=1,2, n=1,2$, will contain, as elements, the derivatives of each differential equation with respect to each variable, duly evaluated in the steady-state. In matrix $J_{m n}$, one finds the derivatives of the state equations $(m=1)$ or of the co-state equations $(m=2)$ with respect to each state variable $(n=1)$ or co-state variable $(n=2)$.

The computation of the elements of the Jacobian matrix will allow for the characterization of the stability properties of the dynamic system.

Proposition 3. The DRAP, when evaluated in the steady-state vicinity, delivers a saddle-path stable equilibrium, with the number of stable trajectories equal to the number of productive tasks (i.e., the number of firms).

Proof. Local stability is approachable by computing the eigenvalues of the Jacobian matrix. Stable and unstable dimensions have correspondence on the signs of the eigenvalues. Specifically, to each negative (positive) eigenvalue, it corresponds a stable (unstable) dimension of the model.

Note that the Jacobian matrix is a square matrix of order equal to twice the number of firms or productive tasks. The Jacobian matrix will contain a number of eigenvalues equal to the matrix order. These eigenvalues are straightforward to compute because $J_{21}$ is a matrix of zeros. ${ }^{5}$ In this case, the eigenvalues of $J$ are necessarily the eigenvalues of matrices $J_{11}$ and $J_{22}$; hence, the contents of $J_{12}$ are not relevant for the purpose at hand, and one does not need to calculate them.

\footnotetext{
${ }^{5}$ Equations relating the co-state variables depend on this variables alone, and not on the state variables.
}

Matrix $J_{11}$ has, as elements in the main diagonal, the following,

$\tilde{j}_{i i}=-\frac{1-\int_{j \in N\{i\}} \omega_{j}^{*} d j}{1-\int_{j \in N} \omega_{j}^{*} d j} \zeta$.

Outside the main diagonal, the elements are

$\tilde{j}_{i \ell}=-\frac{\omega_{i}^{*}}{1-\int_{j \in N} \omega_{j}^{*} d j} \zeta$.

Relatively to matrix $J_{22}$, the respective elements are, in the main diagonal,

$\tilde{j}_{i i}=\rho+\frac{1-\int_{j \in N \backslash\{i\}} \omega_{j}^{*} d j}{1-\int_{j \in N} \omega_{j}^{*} d j} \zeta$.

Outside the main diagonal of $J_{22}$,

$\tilde{j}_{i \ell}=-\frac{\omega_{\ell}^{*}}{1-\int_{j \in N} \omega_{j}^{*} d j} \zeta$.

For matrix $J_{11}$, one of the eigenvalues is

$$
\lambda_{i}=-\frac{1}{1-\int_{j \in N} \omega_{j}^{*} d j} \zeta .
$$

All the others are identical,

$\lambda_{j}=-\zeta, j \in N\{i\}$

A similar result is obtained for $J_{22}$,

$\lambda_{i}=\rho+\frac{1}{1-\int_{j \in N} \omega_{j}^{*} d j} \zeta$

and

$\lambda_{j}=\rho+\zeta, j \in N\{i\}$.

Direct observation indicates that the eigenvalues of $J_{11}$ are all negative and that the eigenvalues of $J_{22}$ are all positive. Therefore, the system is saddle-path stable with stable and unstable spaces possessing an identical dimension. The dimensionality of both the stable and the unstable trajectories is equal to the number of firms that compete for the investment resources. Therefore, there exists a saddle-point equilibrium, with this point having a dimensionality equal to the number of firms.

To address transitional dynamics, one needs to compute the analytical expressions of the stable trajectories through which the endogenous variables converge to the long-term equilibrium point. A first relevant result involves the (absence of) motion of the co-state variable.

Proposition 4. In the convergence towards the steady-state, the costate variable assumes the constant value $p_{i}(t)=p^{*}$.

Proof. For a given firm $i$, the stable trajectory respecting to the co-state variable $p_{i}(t)$ is given by the following general expression,

$p_{i}(t)-p^{*}=\int_{j \in N}\left\{\left(\int_{\ell \in N} \chi_{\ell i}^{p} \chi_{j \ell}^{\omega,-1} d \ell\right)\left[\omega_{j}(t)-\omega_{j}^{*}\right]\right\} d j, i \in N$ 
where $\chi_{\ell i}^{p}$ and $\chi_{j \ell}^{\omega,-1}$ are elements of the matrices $\Xi_{p}$ and $\Xi_{\omega}^{-1}$. Matrix $\Xi_{p}$ is the sub-matrix of the matrix of eigenvectors of the Jacobian $J$ that is associated to the negative eigenvalues and that respects to the co-state variables; $\Xi_{\omega}^{-1}$ is the inverse of the sub-matrix of the same matrix of eigenvectors that respects to the state variables. The matrix of eigenvectors concerning negative eigenvalues is $\Xi=\left[\begin{array}{ll}\Xi_{\omega} & \Xi_{p}\end{array}\right]^{\prime}$.

Taking into consideration the eigenvalues of $J$, the following outcome is obtained: $\Xi_{p}=\overline{0}$, i.e., all elements $\chi_{\ell i}^{p}$ are null values. This implies that expression (21) reduces to $p_{i}(t)=p^{*}, \forall i \in N$, meaning that in the convergence to the steady-state following the stable trajectory, the co-state variable is initially placed at point $p_{i 0}=p^{*}$ and remains there as variable $\omega_{i}(t)$ converges towards its long-term value.

The result in Proposition 4 simplifies significantly the analysis of the DRAP, since one can focus solely on the movement of $\omega_{i}(t)$. In the adjustment to the steady-state, Eq. (4) becomes a linear equation,

$\dot{\omega}_{i}(t)=B^{1 /(1-\beta)}\left(\beta q_{i} p^{*}\right)^{\beta /(1-\beta)}[1-\Omega(t)]-\zeta \omega_{i}(t), \quad i \in N$

that, under steady-state conditions, is equivalent to

$\dot{\omega}_{i}(t)=\frac{\zeta \omega_{i}^{*}}{1-\Omega^{*}}[1-\Omega(t)]-\zeta \omega_{i}(t), \quad i \in N$.

The solution of Eq. (23) for the set of all productive tasks $i$ is,

$\boldsymbol{\omega}=\boldsymbol{\omega}^{*}+\Xi_{\omega} \exp (\Lambda t) \Xi_{\omega}^{-1}\left(\boldsymbol{\omega}_{0}-\boldsymbol{\omega}^{*}\right)$.

In expression (24), $\boldsymbol{\omega}$ is the vector of state variables $\omega_{i}(t), i \in N ; \omega_{0}$ and $\boldsymbol{\omega}^{*}$ are the respective vectors of initial values and steady-state values. Matrix $\Xi_{\omega}$ is the matrix of eigenvectors of $J_{11}$ and $\Xi_{\omega}^{-1}$ its inverse, as defined above; $\Lambda$ is the Jordan matrix of the system, i.e., it is a matrix with the system's eigenvalues in the main diagonal and zeros outside the main diagonal.

Proceeding with the respective computation, the system's solution for each share $\omega_{i}(t)$ is derived,

$\begin{aligned} \omega_{i}(t)= & \omega_{i}^{*}+\frac{\omega_{i}^{*}}{\Omega^{*}}\left[\exp \left(-\frac{\zeta}{1-\Omega^{*}} t\right)-\exp (-\zeta t)\right] \\ & +\left(\omega_{i 0}-\omega_{i}^{*}\right) \exp (-\zeta t), \quad i \in N .\end{aligned}$

The sum of $\omega_{i}(t)$ across the investment shares of all firms delivers the trajectory in time of the aggregate investment share $\Omega(t){ }^{6}$

$\Omega(t)=\Omega^{*}+\left(\Omega_{0}-\Omega^{*}\right) \exp \left(-\frac{\zeta}{1-\Omega^{*}} t\right)$.

Next, one proves that the first-order conditions in the proof of Proposition 1 effectively are the relevant optimality conditions of the problem, under the assumptions that were mentioned in the proof of Proposition 1.

Proposition 5. Let $\Omega_{0}<\Omega^{*}, p(t)=p^{*}$ and

$p^{*}:\left\{\begin{array}{c}\frac{2 \beta-1}{\beta} \frac{1}{\rho+\zeta} \leq p^{*}<\frac{1}{\rho+\zeta} \text { if } \beta>\frac{1}{2} \\ 0<p^{*}<\frac{1}{\rho+\zeta} \text { if } \beta \leq \frac{1}{2} .\end{array}\right.$

Under these conditions, constraints on variables $\omega_{i}(t) \geq 0, \phi_{i}(t) \geq 0$, and $\Omega(t) \leq 1, \Phi(t) \leq 1$ are satisfied, $\forall t \geq 0$.

Proof. The initial value and the steady-state value of $\Omega(t)$ are such that $0<\Omega_{0}<\Omega^{*}<1$. Eq. (26) implies $\Omega(t)=\Omega_{0}$ for $t=0, \Omega(t)=\Omega^{*}$ when

\footnotetext{
${ }^{6}$ The trajectory of the aggregate investment share (Eq. (26)) could be obtained, as well, through the determination of the solution of the scalar differential equation respecting $\Omega(t)$, which is $\dot{\Omega}(t)=-\frac{\zeta}{1-\Omega^{*}}\left[\Omega(t)-\Omega^{*}\right]$. This differential equation is obtained directly from the definition $\dot{\Omega}(t) \equiv \int_{i \in N} \dot{\omega}_{i}(t) d i$, with $\dot{\omega}_{i}(t)$ given by Eq. (23).
}

$t \rightarrow+\infty$, and $\Omega_{0}<\Omega(t)<\Omega^{*}$, for $0<t<+\infty$. Therefore, it is not possible to have any value of the aggregate investment share above 1 ; furthermore, Eq. (25) indicates that $\omega_{i}(t)$ must be a non-negative value, independently of the assumed time moment; to confirm this outcome, note that $\Omega_{0}<\Omega^{*}$ by assumption and that $\exp \left(-\frac{\zeta}{1-\Omega^{*}} t\right)<\exp (-\zeta t)$ for any $t$, thus making the second term of the expression positive; because the sum of the remaining terms, $\omega_{i}^{*}+\left(\omega_{i 0}-\omega_{i}^{*}\right) \exp (-\zeta t)$ must be positive as well, regardless of $t$, one also confirms the veracity of $\omega_{i}(t) \geq 0$.

Under $p_{i}(t)=p^{*}$, expression Eq. (7) is equivalent to

$\phi_{i}(t)=q_{i}^{\beta /(1-\beta)}\left(\beta B p^{*}\right)^{1 /(1-\beta)}[1-\Omega(t)], i \in N$.

The control variable is, according to Eq. (27), a non-negative value. Finally, the sum of $\phi_{i}(t)$ as presented in Eq. (27), and given the steadystate relations, takes the form

$\Phi(t)=\frac{\beta}{1-\beta}\left[1-(\rho+\zeta) p^{*}\right][1-\Omega(t)]$.

In Eq. (28), $\Omega(t)$ may assume any value between 0 and 1 . Therefore, condition $\Phi(t) \leq 1$ is guaranteed whenever $\frac{\beta}{1-\beta}\left[1-(\rho+\zeta) p^{*}\right] \leq 1$. Solving the inequality with respect to $p^{*}$, it yields $p^{*} \geq \frac{2 \beta-1}{\beta} \frac{1}{\rho+\zeta}$. If $\beta \leq 1 / 2$, the constraint is necessarily satisfied, because of the non-negativity of $p^{*}$; the condition must be imposed in the opposite case, i.e., $\beta>1 / 2$.

Eq. (28) can be used to depict graphically the stability trajectory that is followed together by the endogenous aggregate variables of the system. Fig. 2 displays the relation,

Fig. 2 indicates that variables $\Omega(t)$ and $\Phi(t)$ evolve in opposite directions as they converge to the steady-state. Under assumption $\Omega_{0}<\Omega^{*}$, the share of allocated investment increases following the path given by Eq. (26); simultaneously, the overall signaling costs of the whole universe of firms will fall towards the respective steady-state as time unfolds.

\section{The impact of parameter changes}

Two parameters acquire special relevance in the analysis: the investor's sentiment $(B)$ and the quality of the proposals presented by the firms to the investor $\left(q_{i}\right)$. These variables have a same qualitative impact on the value of $\Omega^{*}$, since they influence the matching process between principal and agent in a similar way.

Proposition 6. A positive change on the investor's sentiment or on the quality of any of the project proposals will, ceteris paribus, increase the allocation efficiency of the investment, i.e., it will increase the value of $\Omega^{*}$, placing it closer to 1 .

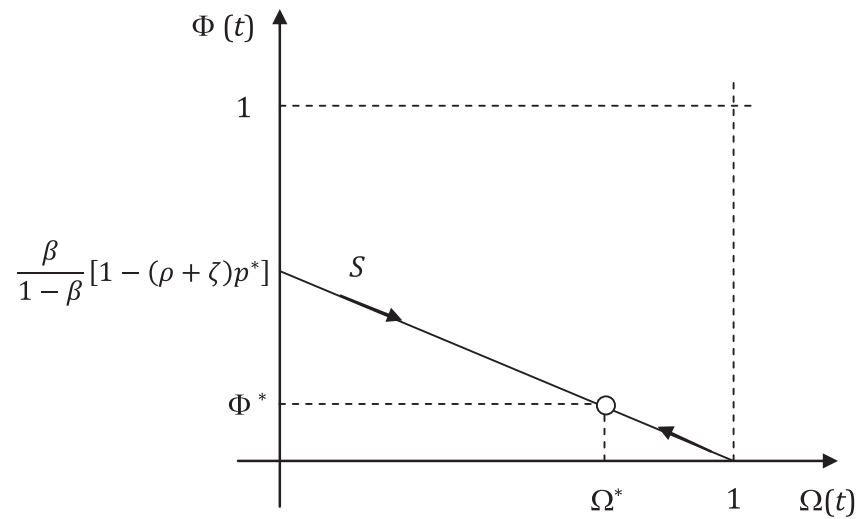

Fig. 2. Saddle-path trajectory for the pair of variables $\Omega(t), \Phi(t)$. 
Proof. Recover the steady-state value of $\Omega(t)$ in expression Eq. (17). The differentiation of this expression with respect to $B$ or $q_{i}$ yields

$\frac{\partial \Omega^{*}}{\partial B}=-\frac{(1-\beta) \zeta}{\left[1-(\rho+\beta \zeta) p^{*}\right]^{2}} \frac{\partial p^{*}}{\partial B}$

or

$\frac{\partial \Omega^{*}}{\partial q_{i}}=-\frac{(1-\beta) \zeta}{\left[1-(\rho+\beta \zeta) p^{*}\right]^{2}} \frac{\partial p^{*}}{\partial q_{i}}$

Since a general explicit expression for $p^{*}$ was not determined, one cannot derive $\frac{\partial p^{*}}{\partial B}$ and $\frac{\partial p^{*}}{\partial q_{i}}$ explicitly. However, a qualitative evaluation of the signs of these derivatives is possible. Returning to equilibrium equality Eq. (11), one observes that a change on $B$ or $q_{i}$ will not exert influence over the rhs expression of the equation. Relatively to the lhs, a positive variation on the value of any of the two parameters will turn the slope of the respective function more pronounced, making the $l h s$ and the rhs to intersect at a lower value of $p^{*}$, as depicted in Fig. 3.

The figure reveals that $\frac{\partial p^{*}}{\partial B}<0$ and $\frac{\partial p^{*}}{\partial q_{i}}<0$ and, therefore, one concludes that a positive sentiment change on the quality of any of the projects presented to the investor will improve the efficiency of the resource allocation: $\frac{\partial \Omega^{*}}{\partial B}>0, \frac{\partial \Omega^{*}}{\partial q_{i}}>0$.

Other two parameters have a substantive influence over the value of $\Omega^{*}$. These are the intertemporal discount rate $(\rho)$ and the percentage of the allocated budget that the investor recovers in each period $(\zeta)$. For these parameters, the following results apply,

Proposition 7. A positive change on the values of parameters $\rho$ and $\zeta$ have a qualitatively ambiguous effect over $\Omega^{*}$.

Proof. Start by noticing that a positive change on $\rho$ or $\zeta$ will imply a reduction on the steady-state value of the co-state variable. This occurs because in the relation between the lhs and the rhs of Eq. (11), the first does not suffer any change as a result of varying $\rho$ or $\zeta$, while the rhs will rotate inward for higher values of each of the two parameters. Fig. 4 clarifies this point.

To evaluate the effect that the change in any of the parameters under consideration exerts on $\Omega^{*}$, one computes the derivatives of Eq. (17) with respect to each of the two parameters,

$$
\begin{aligned}
& \frac{\partial \Omega^{*}}{\partial \rho}=-\frac{(1-\beta) \zeta}{\left[1-(\rho+\beta \zeta) p^{*}\right]^{2}}\left(\frac{\partial p^{*}}{\partial \rho}+\left(p^{*}\right)^{2}\right) \\
& \frac{\partial \Omega^{*}}{\partial \zeta}=-\frac{(1-\beta) \zeta}{\left[1-(\rho+\beta \zeta) p^{*}\right]^{2}}\left(\frac{\partial p^{*}}{\partial \zeta}+\frac{1-\rho p^{*}}{\zeta} p^{*}\right) .
\end{aligned}
$$

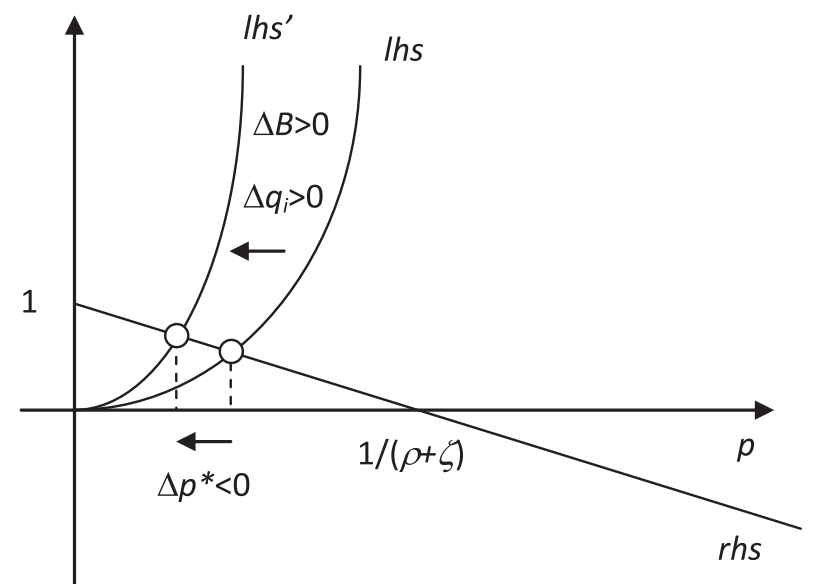

Fig. 3. Effect of a change on $B$ or $q_{i}$ over $p^{*}$.

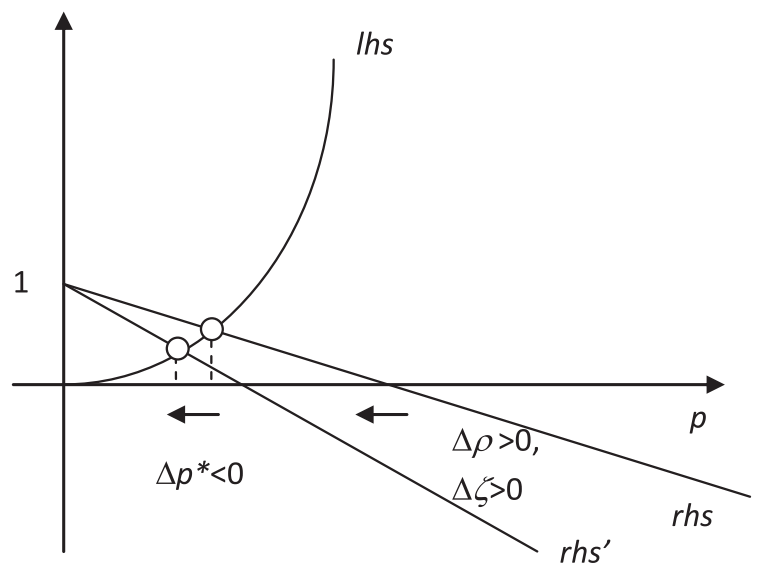

Fig. 4. Effect of a change on $\rho$ or $\zeta$ over $p^{*}$.

Since the signs of terms $\frac{\partial p^{*}}{\partial \rho}+\left(p^{*}\right)^{2}$ and $\frac{\partial p^{*}}{\partial \zeta}+\frac{1-\rho p^{*}}{\zeta} p^{*}$ are not determinable for the general version of the model, one cannot conclude on the qualitative impact of changes on $\rho$ and $\zeta$ over $\Omega^{*}$.

To illustrate the potential of the DRAP in explaining investment allocation, consider a numerical example. Let $\beta=0.25, \int_{i \in N} q_{i}^{\beta /(1-\beta)} d i=1$, $B=2, \rho=0.04, \zeta=0.05$. $^{7}$ For these values, Eq. (11) indicates that the steady-state level of the co-state variable is, approximately, $p^{*}=0.8279$. Taking Eqs. (17) and (20), one also determines the steady-state of the aggregate state and control variables: $\Omega^{*}=0.9675$ and $\Phi^{*}=0.01$. In this case, for the assumed parameter values, $96.75 \%$ of the available investment resources are effectively allocated, in the long-run, to the productive activities and, on the aggregate, firms will need to spend $1 \%$ of the available resources to attain this outcome.

The stable path between $\Omega(t)$ and $\Phi(t)$ is, following (28), $\Phi(t)=$ $0.3085[1-\Omega(t)]$. The stable trajectory, for the specific example under appreciation, is displayed in Fig. 5 .

Under the assumption $\Omega_{0}<\Omega^{*}$, only the segment $0 \leq \Omega(t) \leq 0.9675$, in Fig. 5 , matters. In this segment, a one unit positive change on the aggregate investment share implies a decrease in the value of the aggregate control variable on an amount 0.3085 , as the convergence to the steady-state takes place.

The example might be employed as well to quantitatively assess the impact of changes on parameter values and to confirm the general results presented earlier in this section. Consider first a positive change on the sentiment value, $\Delta B=0.05$. Recomputing the steady-state level of the co-state variable, one obtains $p_{(B \text { new })}^{*}=0.8088$. Hence, the change on $p^{*}$ with origin in the positive sentiment evolution is $\Delta p^{*}=-0.0191$. Resorting to expression Eq. (17), one verifies that $\Omega_{(B \text { new })}^{*}=0.9683$ and, thus, $\Delta \Omega^{*}=0.0008$, i.e., the allocation of resources as improved in 0.8 percentage points.

Consider, in a second moment, a change on the intertemporal discount rate. Let $\Delta \rho=-0.02$. In this case, firms become more patient in the way they evaluate future outcomes. Now, $p_{(\rho \text { new })}^{*}=0.8385$. As discussed earlier, a decrease in $\rho$ must translate on a higher value of the co-state variable steady-state level; in this case, $\Delta p^{*}=0.0106$. Replacing, in Eq. (17), the new values of $\rho$ and $p^{*}$, one obtains the disturbed steady-state level of the aggregate investment share, $\Omega_{(\rho \text { new })}^{*}=0.9677$. For the assumed parameterization, the negative change on the discount rate has provoked a slight positive variation on $\Omega^{*}, \Delta \Omega^{*}=$ 0.0002 ; more patience is, in this case, synonymous of an environment capable of generating a more efficient long-term allocation of resources.

7 The values used in the example are adopted with the objective of fulfilling two criteria: to be empirically plausible and to deliver sensible investment allocation and growth outcomes. Note, however, that some of the parameters are hard to measure and that they can be presented in different scales, as it is the case of the sentiment index, the quality of projects or the matching elasticity. 


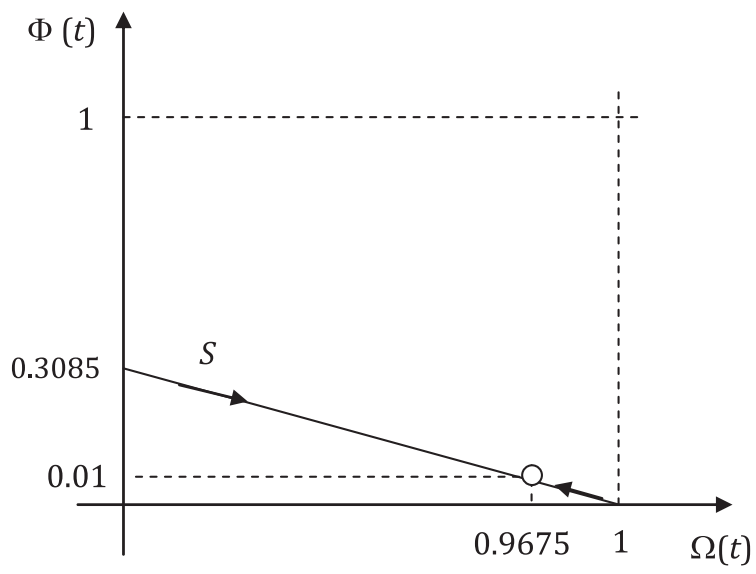

Fig. 5. Illustration of the saddle-path trajectory.

\section{Neoclassical growth under inefficient investment allocation}

In this section, the DRAP, as previously examined, is applied to the standard neoclassical growth model. One builds a Ramsey-CassKoopmans (RCK) optimal control problem where the possibility of inefficient investment allocation is considered.

Definition 4. RCK/DRAP growth model

Consider a representative agent for whom the objective function is

$V(0)=\operatorname{Max}_{c(t)} \int_{0}^{+\infty} u[c(t)] \exp (-\rho t) d t$

In Eq. (33), $c(t) \geq 0$ represents consumption and $u($.$) is the utility$ function. The same intertemporal discount rate $\rho$ as for the DRAP problem is assumed. Optimal control problem (Eq. (33)) is subject to the capital accumulation constraint

$\dot{k}(t)=[f(k(t))-c(t)] \Omega(t)-\delta k(t), k(0)=k_{0}$ given.

Variable $k(t) \geq 0$ represents physical capital per unit of labor, $\delta \in(0,1)$ is the depreciation rate of capital and $f(k(t))$ is the production function. $V(0)$, in Eq. (33), subject to Eq. (34), is the RCK/DRAP growth model.

Observe that the single difference between the RCK/DRAP model and the original formulation of the RCK growth model is the presence of $\Omega(t)$ in the expression of the resource constraint. Instead of considering a measure of potential gross investment, which corresponds to the difference between output, given by the production function, and consumption, effective gross investment is assumed; this relates to the potential level times the aggregate investment share $\Omega(t)$, a variable for which the respective dynamics were approached in previous sections. The remaining share, $[f(k(t))-c(t)][1-\Omega(t)]$ should be interpreted as inefficient investment, i.e., investment with zero return, since, as discussed, it has no productive end.

As it is common in growth models, and in order to facilitate the analytical exploration of the underlying dynamics, let us consider a typical constant intertemporal elasticity of substitution utility function, $U[c(t)]=\frac{c(t)^{1-\theta}-1}{1-\theta}, \theta \in(0,+\infty) \mid\{1\}$, with $1 / \theta$ the intertemporal elasticity of substitution for consumption; assume, as well, a standard CobbDouglas production function, $f(k(t))=A k(t)^{\alpha}$, with $A>0$ the state of technology and $\alpha \in(0,1)$ the output-capital elasticity.

In what follows, the neoclassical growth model is solved for the effective gross investment version and the main results are discussed.
Proposition 8. The optimal trajectory of consumption is, on the RCK/DRAP growth model, given by equation

$$
\begin{aligned}
\dot{c}(t)= & \frac{1}{\theta}\left\{\alpha A k(t)^{-(1-\alpha)} \Omega(t)-B^{1 /(1-\beta)} \beta^{\beta /(1-\beta)} \int_{i \in N}\left[q_{i} p_{i}(t)\right]^{\beta /(1-\beta)} d i\right. \\
& \left.\times \frac{1-\Omega(t)}{\Omega(t)}-(\rho+\delta)+\zeta\right\} c(t) .
\end{aligned}
$$

Proof. The current-value Hamiltonian function of this problem is,

$H[k(t), c(t), \tilde{p}(t)]=\frac{c(t)^{1-\theta}-1}{1-\theta}+\tilde{p}(t)\left\{\left[A k(t)^{\alpha}-c(t)\right] \Omega(t)-\delta k(t)\right\}$

with $\tilde{p}(t)$ the co-state variable associated with $k(t)$. First-order conditions are

$\frac{\partial H}{\partial c(t)}=0 \Rightarrow c(t)^{-\theta}=\tilde{p}(t) \Omega(t)$

$\dot{\tilde{p}}(t)=\rho \tilde{p}(t)-\frac{\partial H}{\partial k(t)} \Rightarrow$

$\dot{\tilde{p}}(t)=(\rho+\delta) \tilde{p}(t)-\alpha A \tilde{p}(t) k(t)^{-(1-\alpha)} \Omega(t)$

and the transversality condition,

$\lim _{t \rightarrow+\infty} \tilde{p}(t) \exp (-\rho t) k(t)=0$.

The differentiation of Eq. (37) with respect to time yields

$-\theta \frac{\dot{c}(t)}{c(t)}=\frac{\dot{\tilde{p}}(t)}{\tilde{p}(t)}+\frac{\dot{\Omega}(t)}{\Omega(t)}$

Growth rate $\frac{\dot{\tilde{p}}(t)}{\tilde{p}(t)}$ is obtainable from Eq. (38), while $\frac{\dot{\Omega}(t)}{\Omega(t)}$ corresponds to the aggregate version of Eq. (4). Replacing these two growth rates in Eq. (40), the rule of motion for consumption, Eq. (35), is obtained.

Because the DRAP was solved independently of the growth model, one may assume that the respective stable trajectory is followed, i.e., according to Proposition $4, p_{i}(t)=p^{*}$. In this case, Eq. (35) simplifies to

$\dot{c}(t)=\frac{1}{\theta}\left\{\alpha A k(t)^{-(1-\alpha)} \Omega(t)-\zeta \frac{\Omega^{*}}{1-\Omega^{*}} \cdot \frac{1-\Omega(t)}{\Omega(t)}-(\rho+\delta)+\zeta\right\} c(t)$

The dynamic analysis of the RCK/DRAP model is feasible through the evaluation of a three-dimensional system involving the constraint on capital accumulation, Eq. (34), the consumption differential equation, Eq. (14), and the differential equation for $\Omega(t)$, that one can recover from Footnote 3.

To proceed, one defines the steady-state of the RCK/DRAP.

Definition 5. Steady-state of the RCK/DRAP growth model

The steady-state of the RCK/DRAP is the point

$\left(k^{*}, c^{*}, \Omega^{*}\right)=\left\{\left(k^{*}, c^{*}, \Omega^{*}\right): \dot{k}(t)=0, \dot{c}(t)=0, \dot{\Omega}(t)=0\right\}$.

Adopting the above definition, the result in Proposition 9 is accomplished.

Proposition 9. The steady-state of the neoclassical growth model with agency relations in the allocation of investment exists and it is unique. The steady-state values of the capital and of the consumption variables are, respectively,

$k^{*}=k_{P}^{*}\left(\Omega^{*}\right)^{1 /(1-\alpha)} ; c^{*}=c_{P}^{*}\left(\Omega^{*}\right)^{\alpha /(1-\alpha)}$ 
with $k_{P}^{*}$ and $c_{P}^{*}$ representing the long-term potential levels of capital and consumption, i.e., the steady-state levels of the two variables when there is full efficiency in the allocation of investment. The respective values are,

$k_{P}^{*}=\left(\frac{\alpha A}{\rho+\delta}\right)^{1 /(1-\alpha)} ; c_{P}^{*}=\left(\frac{1}{\alpha} \rho+\frac{1-\alpha}{\alpha} \delta\right)\left(\frac{\alpha A}{\rho+\delta}\right)^{1 /(1-\alpha)}$.

Proof. Applying Definition 5 to differential Eqs. (34) and (41), it yields, respectively,

$c^{*}=A\left(k^{*}\right)^{\alpha}-\delta \frac{k^{*}}{\Omega^{*}}$

$k^{*}=\left(\frac{\alpha A \Omega^{*}}{\rho+\delta}\right)^{1 /(1-\alpha)}$.

Result Eq. (43) is the one presented in the proposition for the physical capital variable. Value $c^{*}$ is accomplished by replacing $k^{*}$ in Eq. (43) into Eq. (42),

$c^{*}=\left(\frac{1}{\alpha} \rho+\frac{1-\alpha}{\alpha} \delta\right)\left(\frac{\alpha A}{\rho+\delta}\right)^{1 /(1-\alpha)}\left(\Omega^{*}\right)^{\alpha /(1-\alpha)}$.

The graphical inspection of the steady-state result is undertaken via Fig. 6. In this graphic, it is represented a curve that indicates the position of the growth model steady-state $\left(k^{*}, c^{*}\right)$ for every possible value of $\Omega^{*}$. One visually confirms that the typical result of the neoclassical growth model is a particular case of our more complete structure of analysis, namely the limiting case for which there is a perfect allocation of investment resources.

The concave shape of the line in Fig. 6 points out that less than full efficiency on investment allocation penalizes relatively more the capital stock than the level of consumption, in the steady-state.

To address transitional dynamics in the RCK/DRAP, take Eqs. (34), (41), and the equation for the dynamics of $\Omega(t)$. In the vicinity of the steady-state, a linearized representation of the system under appreciation will be

$$
\begin{aligned}
{\left[\begin{array}{c}
\dot{k}(t) \\
\dot{c}(t) \\
\dot{\Omega}(t)
\end{array}\right]=} & {\left[\begin{array}{ccc}
\rho & -\Omega^{*} & \delta \frac{k^{*}}{\Omega^{*}} \\
-\frac{1-\alpha}{\theta}(\rho+\delta) \frac{c^{*}}{k^{*}} & 0 & \frac{1}{\theta}\left(\rho+\delta+\frac{\zeta}{1-\Omega^{*}}\right) \frac{c^{*}}{\Omega^{*}} \\
0 & 0 & -\frac{\zeta}{1-\Omega^{*}}
\end{array}\right] } \\
& \times\left[\begin{array}{c}
k(t)-k^{*} \\
c(t)-c^{*} \\
\Omega(t)-\Omega^{*}
\end{array}\right] .
\end{aligned}
$$

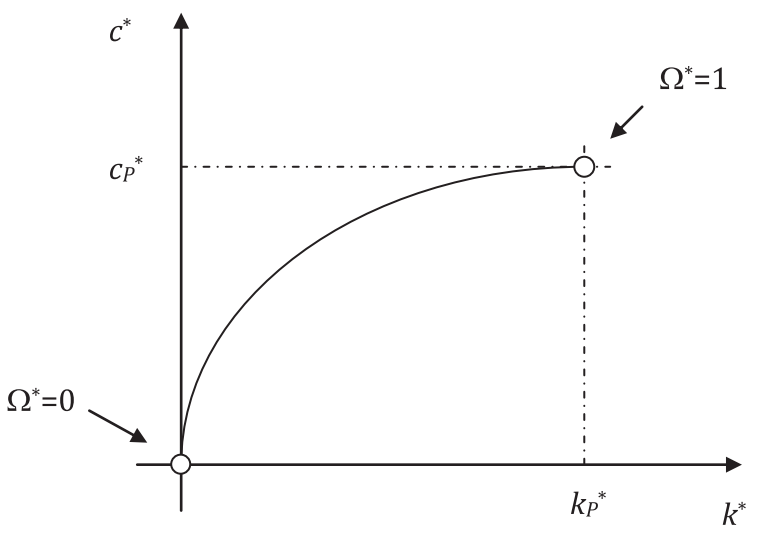

Fig. 6. Steady-state $\left(k^{*}, c^{*}\right)$ for different values of $\Omega^{*}$.
Matricial system Eq. (45) allows for an assessment of the stability properties of the growth model.

Proposition 10. The long-term equilibrium of the RCK/DRAP is saddlepath stable.

Proof. The Jacobian matrix of system Eq. (45) possesses three eigenvalues. One of them relates to the investment share and it confirms the stable nature of the steady-state value $\Omega^{*}$ (this is the eigenvalue $\lambda=$ $\left.-\frac{\zeta}{1-\Omega^{*}}<0\right)$. To evaluate the stability of the growth model, one can concentrate attention on the sub-matrix of the Jacobian relating to capital and consumption variables. The eigenvalues of the respective sub-matrix are such that

$\lambda_{1}^{k, c} \lambda_{2}^{k, c}=-\frac{1-\alpha}{\theta}(\rho+\delta) \frac{c^{*}}{k^{*}} \Omega^{*}<0$

$\lambda_{1}^{k, c}+\lambda_{2}^{k, c}=\rho>0$

The product of the eigenvalues, which corresponds to the determinant of the matrix, is negative, while the sum of the eigenvalues, which is the trace of the Jacobian, takes a positive value. In this case, there will be, necessarily, a positive eigenvalue and a negative eigenvalue, meaning that, as in the original Ramsey model, a saddle-path stable equilibrium prevails.

Let us consider the sub-system of Eq. (45) relating variables $k(t)$ and $c(t)$. Given the relations involving the eigenvalues in the proof of Proposition 10, the sub-system can be written under the form

$\left[\begin{array}{l}\dot{k}(t) \\ \dot{c}(t)\end{array}\right]=\left[\begin{array}{cc}\lambda_{1}^{k, c}+\lambda_{2}^{k, c} & -\Omega^{*} \\ \frac{\lambda_{1}^{k, c} \lambda_{2}^{k, c}}{\Omega^{*}} & 0\end{array}\right]\left[\begin{array}{l}k(t)-k^{*} \\ c(t)-c^{*}\end{array}\right]$

Let $\lambda_{1}^{k, c}$ be the negative eigenvalue and $\lambda_{2}^{k, c}$ the positive eigenvalue. In this case, $\Xi_{k, c}=\left[\begin{array}{ll}\frac{\Omega^{*}}{\lambda_{2}^{k, c}} & 1\end{array}\right]^{\prime}$ is the eigenvector associated to the negative eigenvalue. As a result, the expression of the one-dimensional stable trajectory in the $k(t), c(t)$ locus is

$c(t)-c^{*}=\frac{\lambda_{2}^{k, c}}{\Omega^{*}}\left[k(t)-k^{*}\right]$

The saddle trajectory has a positive slope, meaning that in the adjustment from $\left(k_{0}, c_{0}\right)$ towards $\left(k^{*}, c^{*}\right)$, both variables will exhibit a same qualitative behavior. Again, the full efficiency on the allocation of investment is a particular case of (47), for $\Omega^{*}=1$. Therefore, one concludes that the more efficient the investment allocation is, the less steeper the saddle-path trajectory will be. Under strong inefficiency, a small change on the stock of capital will provoke a significant change on the level of consumption, as the adjustment towards the steadystate unfolds.

Growth dynamics can be graphically evaluated through the construction of the respective phase diagram. The lines for which $\dot{k}(t)=$ 0 and $\dot{c}(t)=0$ are, respectively, $c(t)-c^{*}=\frac{\lambda_{1}^{k, c}+\lambda_{2}^{k, c}}{\Omega^{*}}\left[k(t)-k^{*}\right]$ and $k(t)=$ $k^{*}$. Directional arrows in the four quadrants formed by the lines $\dot{k}(t)=$ 0 and $\dot{c}(t)=0$ are determined by the elements in the first column of the matrix in Eq. (46) and point in the same directions as those in the original Ramsey model phase diagram. ${ }^{8}$ Fig. 7 represents the phase diagram for $\Omega^{*}<1$, making the proper comparison with the benchmark case $\Omega^{*}=1$.

In Fig. 7, one should highlight the difference of position between the saddle-path for $\Omega^{*}<1(S)$ and the saddle-path for $\Omega^{*}=1\left(S_{P}\right)$. They are both positively sloped trajectories, but the first is necessarily steeper

\footnotetext{
${ }^{8}$ See Barro and Sala-i-Martin (2004), chapter 2.
} 
than the second. The position of the steady-state points, in each of the two cases, is represented over the steady-state curve drawn in Fig. 6.

One final appointment in this section goes to the impact of a sentiment shock over the dynamics of capital accumulation and consumption. Intuitively, following the result in Proposition 6, according to which a positive change on the investor's sentiment or confidence will increase the efficiency on the allocation of investment resources to production tasks, it is clear that an upward movement over the line represented in Fig. 6 will occur. This is depicted in Fig. 8.

A discrete shock on the investor's sentiment, as one visualizes in Fig. 8, implies an instantaneous jump from the original steady-state to the saddle-path that is formed with the sentiment disturbance. The immediate adjustment takes place on the value of the control variable, i.e., consumption, and once the new saddle-path is accomplished, both variables, capital and consumption, will follow this stable trajectory until the new steady-state point is reached. Note that in this case there is an instantaneous fall in consumption that comes in the sequence of the positive sentiment shock, given the position of the stable trajectory. The consumption level will then increase with capital to the new steady-state, where both variables will possess higher values than in the pre-shock situation. The short-run fall in consumption might be explained under the idea that a better investor's sentiment implies a reorganization of the productive activity that has its first positive impact over the accumulation of capital, while an increase in the production of consumption goods will occur only in a long-term perspective.

The impact of the sentiment change can be quantified through the computation of short-run and long-run multipliers. Rewrite system Eq. (46) with a vector of exogenous coefficients concerning parameter B,

$$
\left[\begin{array}{l}
\dot{k}(t) \\
\dot{c}(t)
\end{array}\right]=\left[\begin{array}{cc}
\lambda_{1}^{k, c}+\lambda_{2}^{k, c} & -\Omega^{*} \\
\frac{\lambda_{1}^{k, c} \lambda_{2}^{k, c}}{\Omega^{*}} & 0
\end{array}\right]\left[\begin{array}{c}
k(t)-k^{*} \\
c(t)-c^{*}
\end{array}\right]+\left[\begin{array}{c}
\delta \frac{k^{*}}{\Omega^{*}} \frac{\partial \Omega^{*}}{\partial B} \\
\frac{\rho+\delta}{\theta} c^{*} \frac{\partial \Omega^{*}}{\partial B}
\end{array}\right] \Delta B .
$$

Let $J_{k, c}$ be the Jacobian matrix in system Eq. (46). Long-run multipliers are,

$\left[\begin{array}{c}\Delta k(\infty) \\ \Delta c(\infty)\end{array}\right]=-\left(J_{k, c}\right)^{-1}\left[\begin{array}{c}\delta \frac{k^{*}}{\Omega^{*}} \frac{\partial \Omega^{*}}{\partial B} \\ \frac{\rho+\delta}{\theta} c^{*} \frac{\partial \Omega^{*}}{\partial B}\end{array}\right] \Delta B=\left[\begin{array}{c}\frac{k^{*}}{1-\alpha} \frac{\partial \Omega^{*}}{\partial B} \\ \left(\delta+\frac{1}{1-\alpha}\right) k^{*} \frac{\partial \Omega^{*}}{\partial B}\end{array}\right] \Delta B$

Since $\frac{\partial \Omega^{*}}{\partial B}>0$ (see Proposition 6), one confirms the graphical result such that a positive sentiment change generates an increase, in a longterm perspective, both on the stock of accumulated capital and on the steady-state level of consumption.

One can also measure the short-run effect over the consumption variable, since this suffers, in line with what Fig. 8 shows, an initial jump from the first to the second stable trajectory. Assuming that the shock on $B$ is permanent and non-anticipated, the respective multiplier is

$$
\begin{aligned}
\Delta c(0) & =\left[\Delta c(\infty)-\frac{\lambda_{2}^{k, c}}{\Omega^{*}} \Delta k(\infty)\right] \Delta B \\
& =\left[\delta+\frac{1}{1-\alpha}\left(1-\frac{\lambda_{2}^{k, c}}{\Omega^{*}}\right)\right] \frac{k^{*}}{1-\alpha} \frac{\partial \Omega^{*}}{\partial B} \Delta B
\end{aligned}
$$

The sign of Eq. (50) is ambiguous. Because the slope of the new saddle-path, after the positive disturbance on the value of $B$, diminishes, consumption may increase or fall immediately after the shock. In Fig. 8, the consumption level suffers an instantaneous decrease; however,

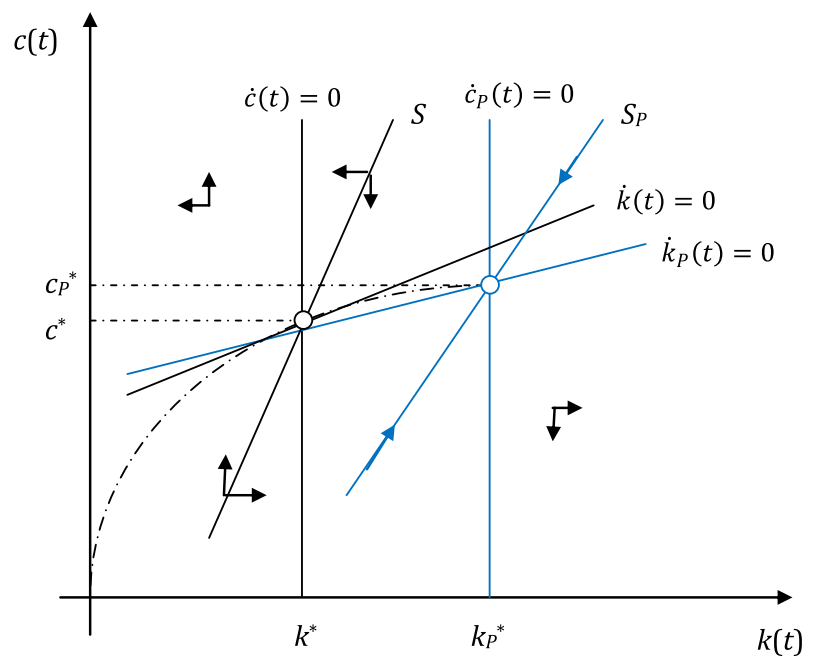

Fig. 7. Phase diagram for the neoclassical growth model under inefficiency on the allocation of investment

different parameter values could generate the opposite result, leading to an outcome as the one illustrated in Fig. 9.

\section{Endogenous growth under inefficient investment allocation}

Consider now an endogenous growth environment,

\section{Definition 6. AK/DRAP growth model}

The problem in Definition 4 acquires the quality of endogenous growth model for an output-capital elasticity equal to 1 .

Assuming $\alpha=1$, the capital accumulation equation is

$\dot{k}(t)=[A \Omega(t)-\delta] k(t)-c(t) \Omega(t), \quad k_{0}$ given

and, taking $p(t)=p^{*}$, the differential equation for the motion of the consumption becomes

$\dot{c}(t)=\frac{1}{\theta}\left[A \Omega(t)-\zeta \frac{\Omega^{*}}{1-\Omega^{*}} \cdot \frac{1-\Omega(t)}{\Omega(t)}-(\rho+\delta)+\zeta\right] c(t)$.

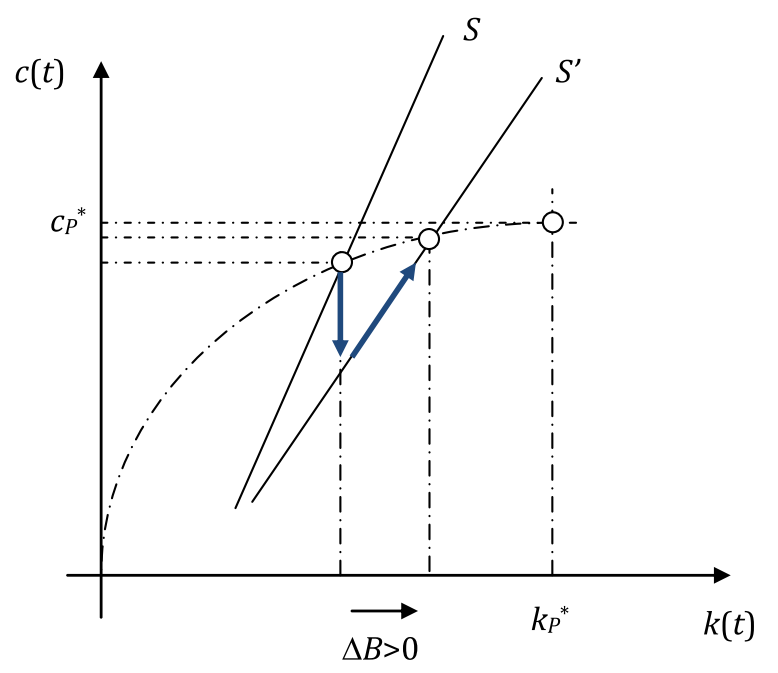

Fig. 8. Effect of a positive investor's sentiment change on the steady-state levels of physical capital and consumption. 
Under endogenous growth, the steady-state acquires new features.

Definition 7. Steady-state in the AK/DRAP model

In the AK/DRAP model, capital and consumption grow at a same positive rate in the steady-state, $\gamma:=\frac{\dot{k}}{k}=\frac{\dot{c}}{c}$. The ratio between consumption and capital must, thus, be constant in the steady-state: for $\psi(t) \equiv \frac{c(t)}{k(t)}$, the steady-state is such that $\psi^{*}=\left\{\psi^{*}: \dot{\psi}(t)=0\right\}$.

\section{Applying Definition 7,}

Proposition 11. In the steady-state of the AK/DRAP model, the ratio consumption-capital is the constant value

$\psi^{*}=\frac{(\theta-1)\left(A \Omega^{*}-\delta\right)+\rho}{\theta \Omega^{*}}$

and capital and consumption grow at rate $\gamma=\frac{1}{\theta}\left[A \Omega^{*}-(\rho+\delta)\right]$. Condition $\Omega^{*}>\frac{\rho+\delta}{A}$ is required to guarantee positive long-term endogenous growth.

Proof. Growth rate $\gamma$, as displayed in the proposition, can be obtained directly from Eq. (52). In fact, $\frac{\dot{c}}{c}=\frac{1}{\theta}\left[A \Omega^{*}-(\rho+\delta)\right]$. The expression of the differential equation for ratio $\psi(t)$ is obtained by noticing that $\frac{\dot{\psi}(t)}{\psi(t)}=$ $\frac{\dot{c}(t)}{c(t)}-\frac{\dot{k}(t)}{k(t)}$. Such expression is

$\dot{\psi}(t)=\left[\frac{1-\theta}{\theta} A \Omega(t)-\frac{1}{\theta} \zeta \frac{\Omega^{*}}{1-\Omega^{*}} \cdot \frac{1-\Omega(t)}{\Omega(t)}-\frac{1}{\theta}(\rho-\zeta)+\frac{\theta-1}{\theta} \delta+\psi(t) \Omega(t)\right] \psi(t)$.

The evaluation of Eq. (54) in the steady-state delivers the value of the long-term consumption-capital ratio in the modified AK model. The result is value Eq. (53) in the proposition. Note that $\psi^{*}$ is necessarily a positive value given the constraint that also guarantees a positive long-term growth rate.

Expression Eq. (53) indicates that in the AK/DRAP model, the consumption-capital ratio in the steady-state depends on the value of $\Omega^{*}$. Observe that $\frac{\partial \psi^{*}}{\partial \Omega^{*}}=\frac{(\theta-1) \delta-\rho}{\theta\left(\Omega^{*}\right)^{2}}$. We identify two cases,

- if $(\theta-1) \delta>\rho$, then $\psi^{*}$ increases with a positive change in $\Omega^{*}$;

- if $(\theta-1) \delta<\rho$, then $\psi^{*}$ decreases with a positive change in $\Omega^{*}$.

The results point to the intuition that a relatively patient agent (low $\rho$ ) will, in the steady-state, see the consumption level increase relatively to the capital stock when the efficiency on the allocation of the

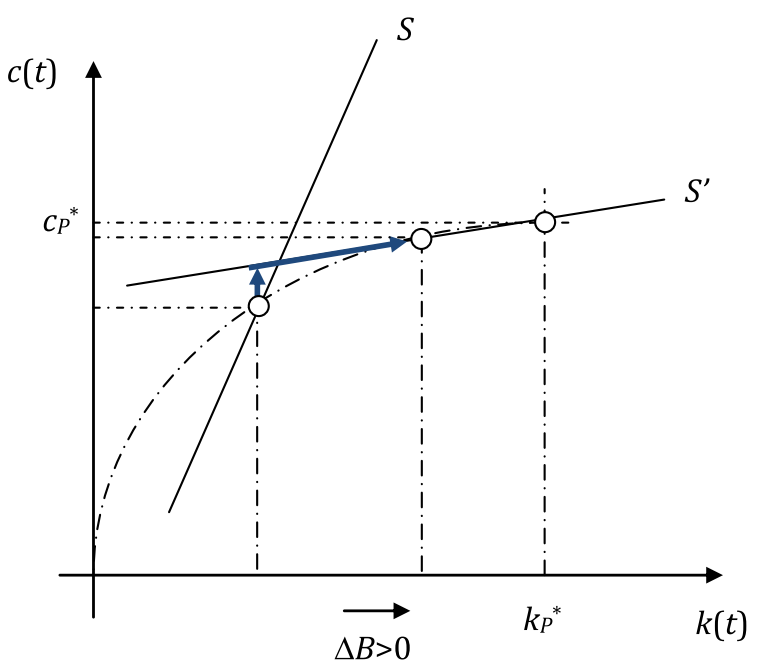

Fig. 9. Effect of a positive investor's sentiment change on the steady-state, without consumption undershooting. investment increases, and vice-versa. Fig. 10 illustrates the steadystate relation between variables $\Omega(t)$ and $\psi(t)$.

Relatively to transitional dynamics, note that in the steady-state vicinity, the AK/DRAP model has correspondence in the following system,

$$
\left[\begin{array}{c}
\dot{\psi}(t) \\
\dot{\Omega}(t)
\end{array}\right]=\left[\begin{array}{cc}
\Omega^{*} \psi^{*} & \left(\psi^{*}\right)^{2}-\frac{\theta-1}{\theta} A \psi^{*}+\frac{\zeta}{\theta} \frac{1}{\left(1-\Omega^{*}\right) \Omega^{*}} \\
0 & -\frac{\zeta}{1-\Omega^{*}}
\end{array}\right]\left[\begin{array}{c}
\psi(t)-\psi^{*} \\
\Omega(t)-\Omega^{*}
\end{array}\right]
$$

Eigenvalues are $\lambda_{1}^{\psi, \Omega}=\Omega^{*} \psi^{*}>0$ and $\lambda_{2}^{\psi, \Omega}=-\frac{\zeta}{1-\Omega^{*}}<0$, and, thus, the steady-state is saddle-path stable. Observe that the motion of $\Omega(t)$ is independent of $\psi(t)$, what implies that, in the convergence towards the steady-state, the consumption-capital ratio is chosen such that it remains undisturbed in the steady-state value $\left(\psi(t)=\psi^{*}\right)$, i.e., only the dynamics of $\Omega(t)$, as mentioned in Section 5 , matters.

A last remark goes to the effect of a sentiment change over the steady-state of the AK/DRAP model. Because $\Delta B>0$ implies $\Delta \Omega^{*}>0$, a positive change on the investor's sentiment triggers a larger steadystate growth rate for the main economic indicators, i.e., capital and consumption. The effect of a change on sentiment over the steadystate growth rate is as much larger as the better is the state of technology and the higher is the intertemporal elasticity of substitution for consumption. Given that $\frac{\partial \psi^{*}}{\partial \Omega^{*}}$ does not have an unequivocal sign one cannot assert, unambiguously, which is the impact of a sentiment change over the consumption-capital steady-state ratio.

\section{Conclusion}

Neoclassical and endogenous growth models offer a compelling explanation on how the economy accumulates physical capital and on which is the consumption trajectory in time that maximizes utility. These models have, as an underlying assumption, the notion that investment is always allocated efficiently and all investment resources are used in production in every time moment. In such a setting, the economy is in a position of full employment of resources.

In this paper, one asks what are the growth implications of assuming an investment allocation process subject to inefficiencies that originate on an agency relation between a representative investor and a large number of firms that compete for financial resources to develop their activities. The investment problem explains how some investment resources are left unused at each time period provoking an inefficiency that will certainly influence the growth process. Assuming inefficient investment allocation, long-term capital and consumption levels will fall below the full efficiency levels, in the neoclassical model, and the long-term growth rate will also be lower than in the benchmark case, when taking the AK endogenous growth model.

The investment allocation problem is designed such that the investor's sentiment plays a relevant role. Sentiments enter the model via the matching between resource demand from firms and resource supply by the investor. An upgrade in the investor's sentiment will increase the efficiency allocation, producing as well a result in terms of growth outcomes closer to what the original Ramsey-Cass-Koopmans and AK models show. This setting is appropriate to study business cycles in the environment typically considered by growth theorists: changes on sentiments provoke changes on growth outcomes.

Future research on the theme of this paper may follow various directions. Three suggestive paths for further inquiry include an explicit consideration of the dynamics of project quality evolution; the design of a network of social contact among firms that may influence the degree of optimism or pessimism of the representative investor; and the adaptation to an open economy scenario where the representative investor may choose to invest domestically or abroad, providing different financing conditions for firms in each location. 


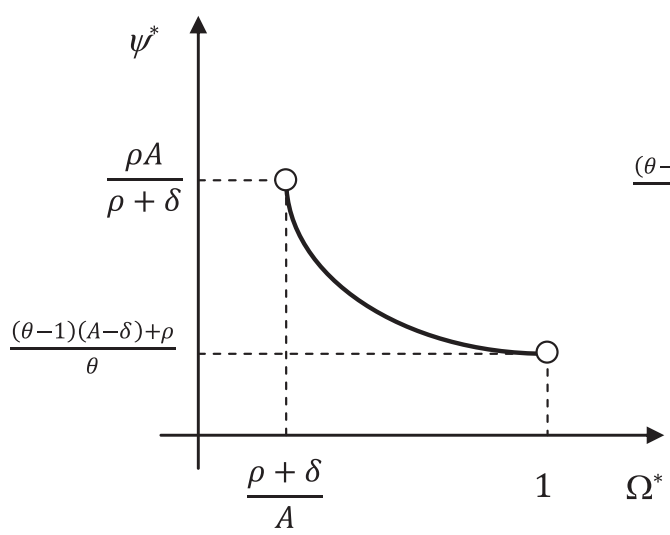

A) $(\theta-1) \delta<\rho$

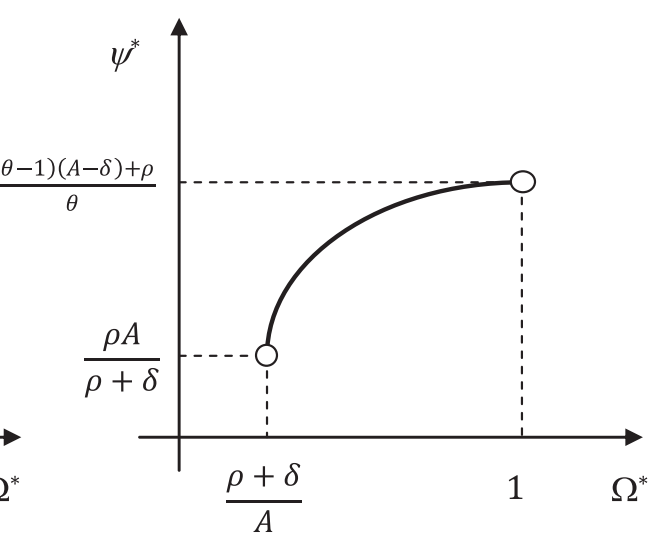

B) $(\theta-1) \delta>\rho$

Fig. 10. Steady-state relation between $\Omega^{*}$ and $\psi^{*}$.

\section{References}

Agliardi, E., Agliardi, R., 2008. Progressive taxation and corporate liquidation policy. Econ. Model. 25, 532-541.

Akerlof, G.A., Shiller, R.J., 2009. Animal Spirits: How Human Psychology Drives the Economy, and Why It Matters for Global Capitalism. Princeton University Press, Princeton, NJ.

Albuquerque, R., Wang, N., 2008. Agency conflicts, investment, and asset pricing. J. Financ. 63, 1-40.

Alfaro, L., Charlton, A., 2007. Growth and the quality of foreign direct investment: is all FDI equal? CEP Discussion Paper No. 0830

Angeletos, G.M., La'O, J., 2013. Sentiments. Econometrica 81, 739-779.

Arif, S., Lee, C.M.C., 2014. Aggregate investment and investor sentiment. Graduate School of Business Research Paper No. 3061. Stanford University.

Azariadis, C., 1981. Self-fulfilling prophecies. J. Econ. Theory 25, 380-396.

Barro, R.J., Sala-i-Martin, X., 2004. Economic Growth. 2nd edition. MIT Press, Cambridge, MA.

Beckaert, G., Harvey, C.R., Lundblad, C., 2011. Financial openness and productivity. World Dev. 39, 1-19.

Benhabib, J., Day, R.H., 1981. Rational choice and erratic behaviour. Rev. Econ. Stud. 48, 459-471.

Benhabib, J., Farmer, R.E.A., 1994. Indeterminacy and increasing returns. J. Econ. Theory 63, 19-41.

Boldrin, M., Nishimura, K., Shigoka, T., Yano, M., 2001. Chaotic equilibrium dynamics in endogenous growth models. J. Econ. Theory 96, 97-132.

Brock, W.A., Hommes, C.H., 1997. A rational route to randomness. Econometrica 65 1059-1095.

Brock, W.A., Hommes, C.H., 1998. Heterogeneous beliefs and routes to chaos in a simple asset pricing model. J. Econ. Dyn. Control. 22, 1235-1274.

Burkart, M., Panunzi, F., 2006. Agency conflicts, ownership concentration, and legal shareholder protection. J. Financ. Intermed. vol. 15, 1-31.

Cass, D., Shell, K., 1983. Do sunspots matter? J. Polit. Econ. 91, 193-227.

Chetty, R., Saez, E., 2010. Dividend and corporate taxation in an agency model of the firm. Am. Econ. J. Econ. Policy 2, 1-31.

Christiano, L., Harrison, S., 1999. Chaos, sunspots and automatic stabilizers. J. Monet. Econ. 44, 3-31.

Day, R.H., 1982. Irregular growth cycles. Am. Econ. Rev. 72, 406-414.

De Grauwe, P., 2011. Animal spirits and monetary policy. Economic Theory 47, 423-457.

Deneckere, R., Pelikan, S., 1986. Competitive chaos. J. Econ. Theory 40, 13-25.

Drugeon, J.P., 2013. On the emergence of competitive equilibrium growth cycles. Economic Theory 52, 397-427.

Eriksson, C., Lindh, T., 2000. Growth cycles with technology shifts and externalities. Econ. Model. 17, 139-170.

Evans, G.W., Honkapohja, S., Romer, P., 1998. Growth cycles. Am. Econ. Rev. 88, 495-515.
Francois, P., Lloyd-Ellis, H., 2003. Animal spirits through creative destruction. Am. Econ. Rev. 93, 530-550.

Gomes, O., 2008. Too much of a good thing: endogenous business cycles generated by bounded technological progress. Econ. Model. 25, 933-945.

Gomes, O., 2014. Agency relations in the brain: towards an optimal control theory. Econ. Bull. 34 (4), 2179-2189.

Gomes, O., 2015. A budget setting problem. In: Bourguignon, J.P., Jelstch, R., Pinto, A.A. Viana, M. (Eds.), Dynamics, Games and Science - International Conference and Advanced School Planet Earth, DGS II. Springer International Publishing, pp. 285-294 (chapter 15).

Grandmont, J.M., 1985. On endogenous competitive business cycles. Econometrica 53 995-1045.

Guerrazzi, M., 2012. The animal spirits hypothesis and the Benhabib-Farmer condition for indeterminacy. Econ. Model. 29, 1489-1497.

Gupta, S., Kangur, A., Papageorgiou, C., Wane, A., 2014. Efficiency-adjusted public capital and growth. World Dev. 57, 164-178.

King, R.G., Rebelo, S.T., 1999. Resuscitating real business cycles. In: Taylor, J.B., Woodford M. (Eds.), Handbook of Macroeconomics vol. 1. Elsevier, pp. 927-1007 (chapter 14).

La Porta, R., Lopez-de-Silanes, F., Shleifer, A., Vishny, R.W., 2000. Agency problems and dividend policies around the world. J. Financ. 55, 1-33.

Matsuyama, K., 1999. Growing through cycles. Econometrica 67, 335-348.

Matsuyama, K., 2001. Growing through cycles in an infinitely lived agent economy J. Econ. Theory 100, 220-234.

Milani, F., 2014. Sentiment and the US business cycle. Department of Economics Working Paper No. 141504. University of California, Irvine.

Montone, M., Zwinkels, R.C.J., 2015. Investor sentiment and employment. Tinbergen Institute Discussion Paper No. 15-046/IV/DSF91.

O'Toole, C.M., Tarp, F., 2014. Corruption and the efficiency of capital investment in developing countries. J. Int. Dev. 26, 567-597.

Rajan, R., Servaes, H., Zingales, L., 2000. The cost of diversity: the diversification discount and inefficient investment. J. Financ. 55, 35-80.

Rebelo, S.T., 2005. Business cycles. Ann. Econ. Financ. 6, 229-250.

Sala-i-Martin, X., Artadi, E.V., 2002. Economic growth and investment in the Arab world. Discussion Paper 0203-08. Columbia University.

Shinagawa, S., 2013. Endogenous fluctuations with procyclical R\&D. Econ. Model. 30 $274-280$.

Shleifer, A., Vishny, R.W., 1989. Management entrenchment: the case of manager-specific investments. J. Financ. Econ. 25, 123-139.

Walde, K., 2002. The economic determinants of technology shocks in a real business cycle model. J. Econ. Dyn. Control. 27, 1-28.

Walde, K., 2005. Endogenous growth cycles. Int. Econ. Rev. 46, 867-894.

Weder, M., 2004. Near-rational expectations in animal spirits models of aggregate fluctuations. Econ. Model. 21, 249-265. 